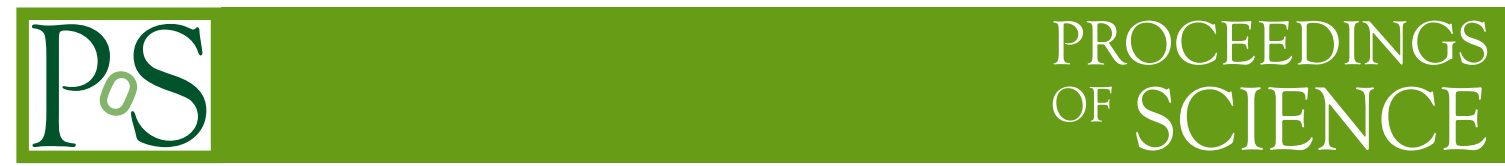

\title{
Status and developments of event generators
}

\author{
Torbjörn Sjöstrand \\ Lund University (SE) \\ E-mail: torbjornethep.lu.se
}

Event generators play a crucial role in the exploration of LHC physics. This presentation summarizes news and plans for the three general-purpose pp generators HERWIG, PYTHIA and SHERPA, as well as briefer notes on a few other generators. Common themes, such as the matching and merging between matrix elements and parton showers, are highlighted. Other topics include a historical introduction, from the Lund perspective, and comments on the role of MCnet.

Fourth Annual Large Hadron Collider Physics

13-18 June 2016

Lund, Sweden 


\section{Introduction}

Event generators have come to play a key role in particle physics, as the bridge that allows direct contact between the theoretical idealized world and the experimental reality. But it is a bridge that does not come for free; it has to be designed, constructed and maintained, and often expanded to meet more demands. In this presentation we will describe the status and development trends among the main generators for pp physics, with main emphasis on the last few years, but with some historical background. A starting platform is provided by the 2011 MCnet review article [1], but there are also relevant later reviews [2, 3, 4, 5]. We also refer to Gavin Salam's presentation for the very important input provided by the explosion of higher-order calculations [6].

To illustrate the diversity of challenges that a full-fledged generator has to face, let us begin with a short overview of the main physics components, figure 1 , most of which will be revisited.

- Initially two hadrons are coming in on a collision course, each with a partonic flux given by the Parton Distribution Functions (PDFs).

- A collision between two partons, one from each side, gives the hard process of interest, characterized by the relevant matrix elements (MEs). These usually are leading order (LO) or next-to-LO (NLO), increasingly also next-to-NLO (NNLO).

- When short-lived "resonances" are produced in the hard process, such as the top, $\mathrm{W}^{ \pm}, \mathrm{Z}^{0}$ or $\mathrm{H}^{0}$, their decay has to be viewed as part of this process itself.

- A collision implies accelerated charges, and thereby bremsstrahlung. This can be described in terms of Parton Showers (PSs), usually split into Initial-State Radiation (ISR) and FinalState Radiation (FSR).

- Since PSs describe the same physics as higher-order MEs do, they must be combined consistently, with PSs adding multiple softer emissions not covered by the fixed-order hard MEs, without gaps or overlaps. This is called matching and merging (M\&M), where matching is the procedure to obtain a smooth transition for a fixed parton multiplicity and merging the combination of several multiplicities, but often the two aspects are intertwined.

- Since the hadrons are made up of a multitude of partons, further parton pairs may collide within one single hadron-hadron collision - MultiParton Interactions (MPIs), each associated with its ISR and FSR.

- Much of the incoming energy and flavours remain in the beam-beam remnants (BBRs), which also carry colours that compensate the colours taken away by the colliding partons.

- Until now, the colour structure has been studied on a local level, and usually bookkept in the $N_{C} \rightarrow \infty$ limit [7], where colour assignments are unambiguous. The overall colour topology may be rearranged relative to the naive picture, colour reconnection (CR), e.g. in a way that reduces the "free energy" in the colour fields.

- As the partons created in the previous steps recede from each other, confinement forces become significant. These colour fields break up into a primary generation of hadrons fragmentation. (Alternatively hadronization, but this can also be used in a more general sense, encompassing most nonperturbative aspects.)

- Many of those primary hadrons are unstable and decay further at various timescales.

- Since many hadrons are produced in close proximity the possibility exists that they scatter 


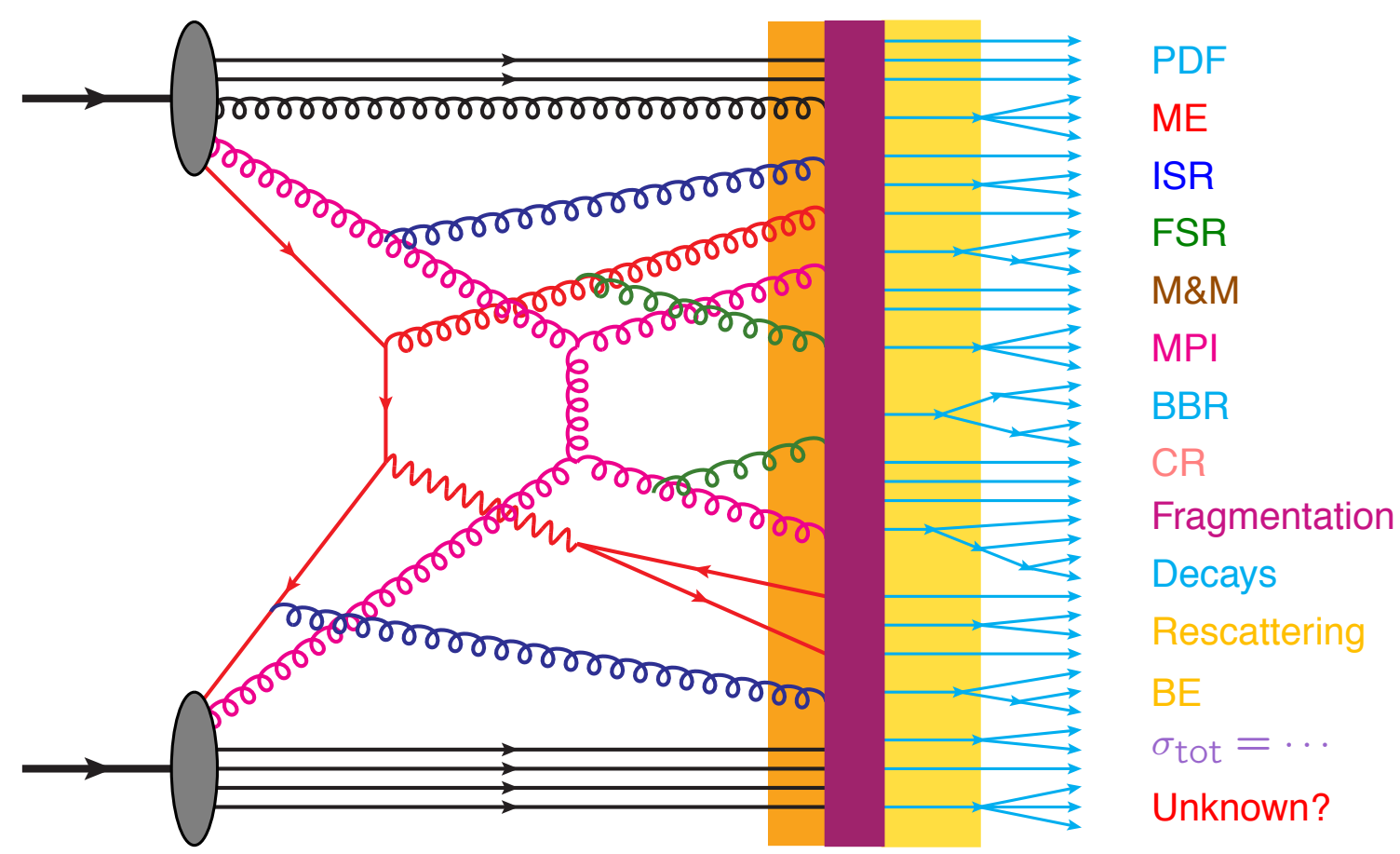

Figure 1: The main components in the generation of an event.

against each other, before or after decays — rescattering.

- For the same proximity reason, there is the potential for significant Bose-Einstein (BE) effects among identical particles.

- So far we have considered events in the context of a hard interaction, but all aspects of the total cross section must be modeled, not least the character of diffractive events as opposed to nondiffractive ones.

- To these points we should add the unknown, e.g. whether some kind of Quark-Gluon Plasma (QGP) is produced also in pp collisions, as hinted by recent experimental observations.

The above points are arranged roughly in order of increasing time scales and decreasing understanding, as we move from perturbative to nonperturbative physics.

This article is organized as follows. Since 2016 marks the 40th anniversary of the Lund QCD phenomenology group, and given the location of this conference, section 2 contains a few historical remarks. Section 3 introduces the three general-purpose generators and the role of MCnet, while sections 4-6 summarizes news on HERWIG, SHERPA and PYTHIA, with some common themes in section 7. A brief overview of some other generators are presented in section 8 , and finally section 9 contains a summary and outlook.

\section{Lund phenomenology flashback}

In 1976, when Bo Andersson and Gösta Gustafson started the Lund group, QCD was still young and poorly understood. The idea of a linear confinement by some stringlike mechanism existed since the late sixties, and linear confinement also had some support in QCD studies. 


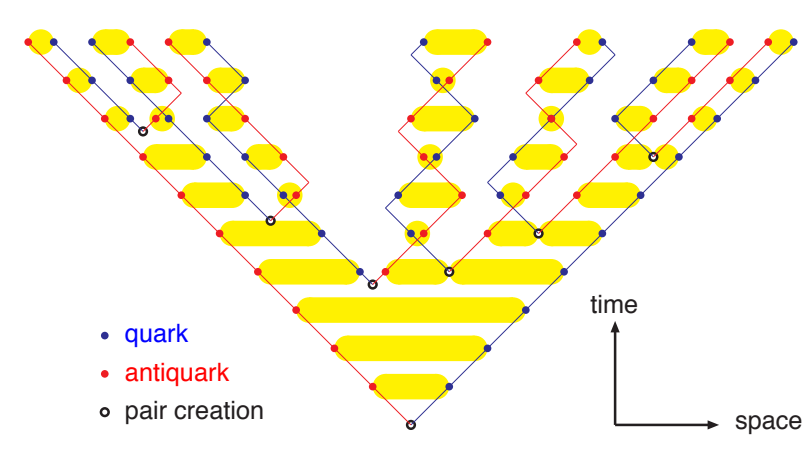

(a)

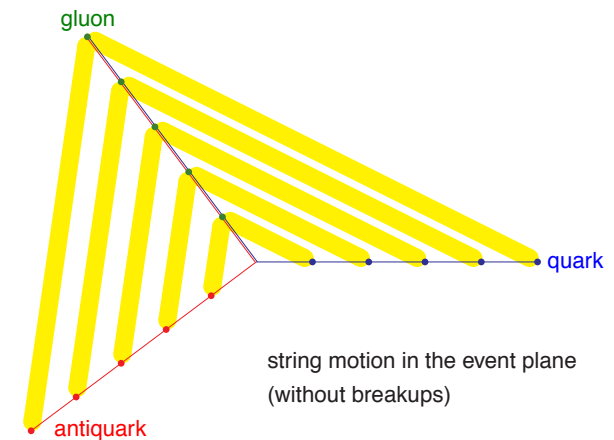

(b)

Figure 2: (a) String breakup in a q $\overline{\mathrm{q}}$ event. (b) String drawing in a $\mathrm{q} \overline{\mathrm{q} g}$ event.

Therefore a straight string (without troublesome transverse vibrations) stretched between a pair of high-energy partons was chosen as a simple and Lorentz-covariant description of a linear potential $V(r)=\kappa r$. The string tension $\kappa \approx 1 \mathrm{GeV} / \mathrm{fm}$ was known from Regge phenomenology. The one-dimensional string is only to be viewed as a simple parametrization of a flux tube with a transverse size of hadronic dimensions, maybe with a structure analogous to a vortex line in a type II superconductor.

A string stretched between an original $\mathrm{q}_{0} \overline{\mathrm{q}}_{0}$ pair can break by the creation of new $\mathrm{q}_{i} \overline{\mathrm{q}}_{i}$ pairs, figure $2 a$. Ordering them $1 \leq i \leq n-1$ from the quark end results in the production of $n$ hadrons $\mathrm{q}_{0} \overline{\mathrm{q}}_{1}, \mathrm{q}_{1} \overline{\mathrm{q}}_{2}, \ldots, \mathrm{q}_{n-1} \overline{\mathrm{q}}_{0}$. The fragmentation begins at the center of the event and then spreads outwards, roughly along a hyperbola of constant proper time. Since the production vertices are spacelike separated, any time ordering is Lorentz frame dependent, however. It is therefore allowed to consider particle production e.g. starting at the $\mathrm{q}_{0}$ end, and recursively split the system into a hadron plus a smaller remainder-system [8].

The 1978 article by Field and Feynman [9] used computer simulation to describe the fragmentation of a single quark. This approach allowed more detailed studies than had been possible by previous analytic methods, and marked the starting point for a more widespread use of computers to provide theory predictions. (A previous study by Artru and Mennessier [10] having passed largely unnoticed.) So also in Lund, where the first public version of the JETSET program provided a setup that could simulate jet fragmentation both according to the Lund prescription and to the Field-Feynman one. It was a Fortran program consisting of roughly 200 punched cards, written under most primitive conditions, but a beginning.

In 1980 the fragmentation model was extended from $q \bar{q}$ to $q \bar{q} g$ events. The crucial step was to apply an $N_{C} \rightarrow \infty$ picture, wherein one string piece was stretched from the $\mathrm{q}$ to the $\mathrm{g}$ and another on from the $\mathrm{g}$ to the $\overline{\mathrm{q}}$, figure $2 b$. The absence of a corresponding direct connection between the $\mathrm{q}$ and $\bar{q}$ led to asymmetries in the particle production where, inside the quark jet, e.g., the rate would be enhanced (depleted) on the side toward the gluon (antiquark) [11]. These predicted features were rapidly confirmed by the JADE collaboration at PETRA [12]. The JETSET code had now grown to around 1000 lines, but was still sent to DESY as a deck of punched cards. Not only did the observation of "the string effect" mean a breakthrough for the Lund model, but it may also have been the first time when a new particle physics idea and the code to help test it were made 
available to the experimental community at the same time. As it happened, the JADE study was done somewhat differently than suggested, highlighting the larger flexibility of a code relative to an analytic prediction.

This approach has caught on, and the generators of today are very much correlated with the development of new physics ideas, be it for parton showers, for matching and merging, for multiparton interactions and colour reconnection, or for hadronization. Conversely, the user choice of a specific generator in a specific setup is an active choice of physics ideology, a point that is not always fully appreciated today, where technical accuracy $\left(\mathrm{N}^{n} \mathrm{LO}\right)$ is at the center of attention. Since often it is not known which is the "right" answer, it is necessary to compare programs and options, and relate observed differences to the underlying physics assumptions.

Over the years, the Lund group has contributed with a number of further new ideas, notably on topics such as

- dipole showers,

- backwards evolution for ISR,

- multiparton interactions,

- colour reconnection,

- the modelling of heavy-ion collisions in the absence of QGP formation,

- matching and merging between matrix elements and parton showers,

- descriptions of small- $x$ evolution, and

- handling of QCD effects in many scenarios for physics Beyond the Standard Model (BSM).

The generator work to match these physics ideas has been continued, most notably with Pythia, which in time subsumed Jetset, but also with Fritiof, ARIADNe, LDC and DiPsy. Former Lund members have written programs like LEPTO, VINCIA and DIRE. And many more programs make use of PYTHIA, such as RAPGAP, HIJING and even GEANT.

\section{The workhorses and MCnet}

There are three general-purpose event generators for LHC pp physics: HERWIG, PYTHIA and SHERPA. They all offer convenient frameworks for a wide range of physics studies, covering essentially all of the aspects listed in the Introduction, but with slightly different historical background and interests.

- Pythia goes back to JeTSET, begun in 1978, in the hadronization studies of the time, and further development has continued to stress nonperturbative physics.

- Herwig is the successor to EARWIG, begun in 1984, and originated in the modelling of coherence effects by an angular-ordered parton shower, which then was complemented by a cluster fragmentation model.

- Sherpa grew out of a matrix element calculator (AMEGIC++) and a parton shower program (APACIC++), begun in 1999, and from the onset stressed the need to match and merge these two techniques consistently.

SHERPA is the only of the three to have been written in $\mathrm{C}++$ from the onset; the other two have had to undergo a time-consuming transition from the original Fortran codes. 


\begin{tabular}{|l|l|}
\hline Program & Homepage \\
\hline HERWIG & https://herwig.hepforge.org/ \\
PYTHIA & http://home.thep.lu.se/Pythia/ \\
SHERPA & https://sherpa.hepforge.org/trac/wiki \\
\hline DIPSY & http://home.thep.lu.se/DIPSY/ \\
DIRE & https://direforpythia.hepforge.org/ \\
HEJ & http://hej.web.cern.ch/HEJ/index.html \\
VINCIA & http://vincia.hepforge.org/ \\
\hline
\end{tabular}

Table 1: Home pages of some of the generators mentioned in this article.

While competitors at some level, the three projects also benefit from each other, and join forces within the EU-funded MCnet network. This network also encompasses a number of other projects, notably the MADGRAPH matrix element generator, but also the ARIADNE, DIPSY and HEJ "plugin" programs to the main generators, and standard general facilities such as RIVET [13], PROFESSOR [14], LHAPDF [15], HEPMC [16] and HepForge [17]. In addition to internal activities, like generator development and $\mathrm{PhD}$ student training, it also offers services to the community. One such is the possibility for $\mathrm{PhD}$ students to come to a node for 3-6 months, to carry out some specific projects of mutual interests. Another is the arrangements of summer schools on event generators, in 2016 at DESY together with CTEQ, in 2017 in Lund, 3-7 July. Both experimentalists and theorists are most welcome!

MCnet has received funding 2007-10, 2013-16, and is now approved for 2017-20. Nodes in the new incarnation are Manchester, Durham, Glasgow, Göttingen, Heidelberg, Karlsruhe, UC London, Louvain and Lund, with CERN, SLAC and Monash (Melbourne) as partners.

\section{HeRwig news}

HERwig 7.0 was released at the end of 2015 [18]. This is a continuation of the HERWIG++ development series [19], begun 16 years earlier, to replace the Fortran HERWIG 6 program with one in $\mathrm{C}++$. This objective is now achieved; the HERWIG++ 3.0 code fully supersedes the HERWIG 6 one, and was therefore renamed Herwig 7.0. It replaces all previous Herwig and HeRwig++ versions.

The main physics news is that NLO matched to parton showers is default for most SM processes. This is fully automated, with no external codes to run separately and no intermediate event files. There is a choice between a subtractive (MC@NLO type [20]) and a multiplicative (PowHeg type [21]) matching. This is made possible by the MAтснвоX module [22], figure 3, which interfaces to a large number of different external providers of tree and one-loop amplitudes. The NLO matching subtractions are then performed internally, using the Catani-Seymour dipole approach [23]. All auxiliary programs, the ME ones as well as general utilities such as LHAPDF and FASTJET [25], can be downloaded, installed and built together with HERWIG with the help of a bootstrap script.

HERWIG now comes with two different shower algorithms, the traditional angular-ordered one [26] and a newer dipole shower [27]. The former has been improved in a number of respects, e.g. 


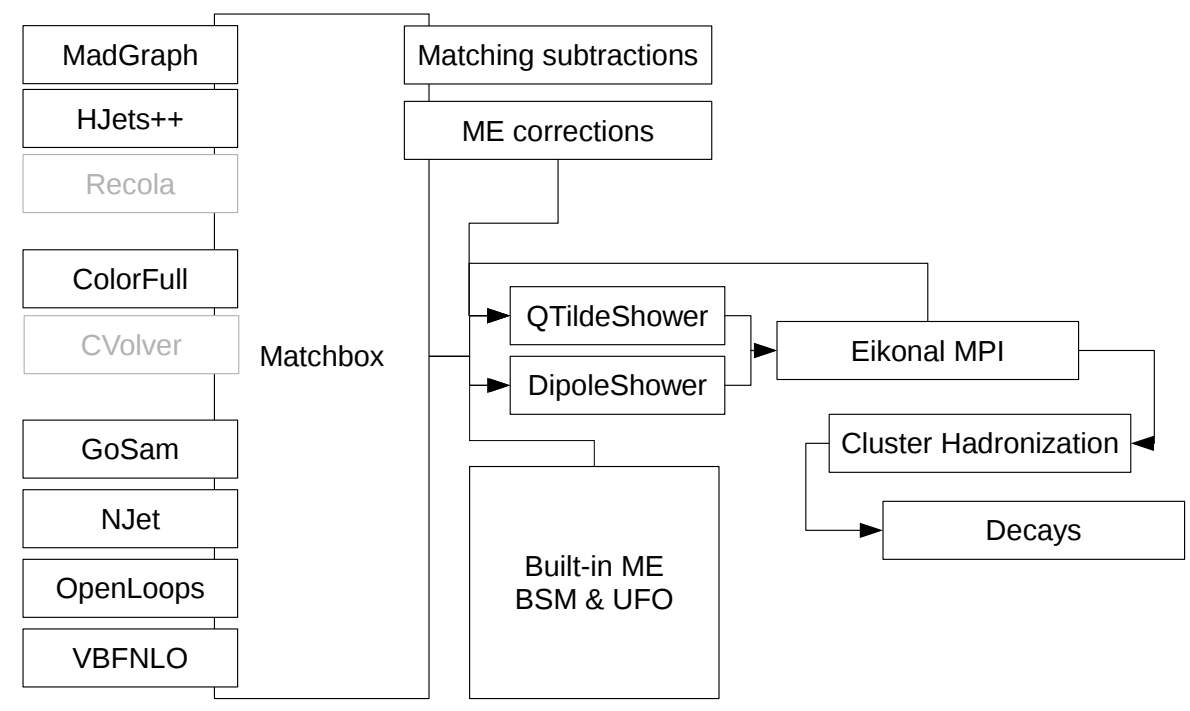

Figure 3: The structure of the HERWIG 7 program, highlighting the role of MATCHBOX [24].

to include QED showering and spin correlations (at LO), and $g \rightarrow q \bar{q}$ branchings are no longer angular-ordered to reflect the absence of a soft singularity in it. Uncertainties from scale variations in the shower can now automatically be evaluated, see further section 7.

Also many other improvements have been made, such as new default tune that includes both minimum-bias and underlying-event data, exemplified in figure 4, or several options to parallelize execution. Last but not least, the documentation of the program has been vastly improved, see the web page for the code, manual, further instructions and lots of auxiliary material.

In the near future the HERWIG 7.1 release will bring NLO multijet merging, based on uni-

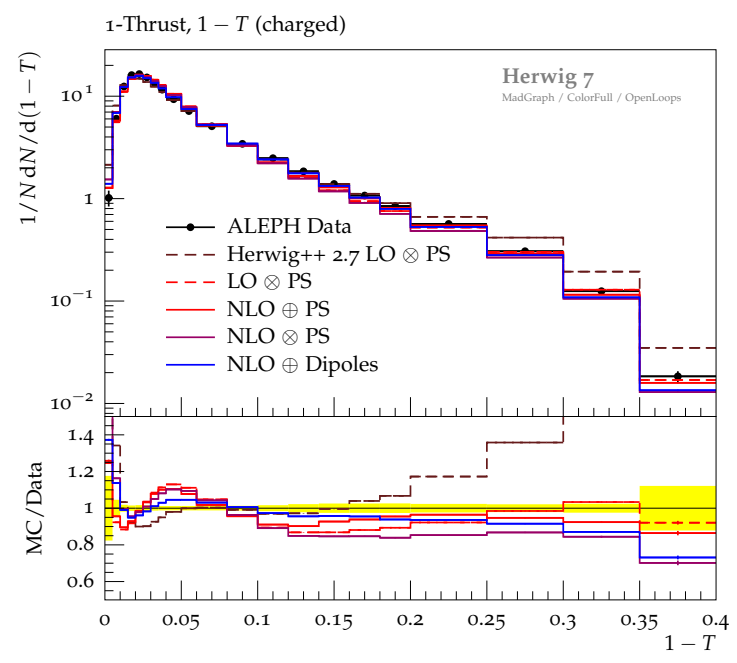

(a)

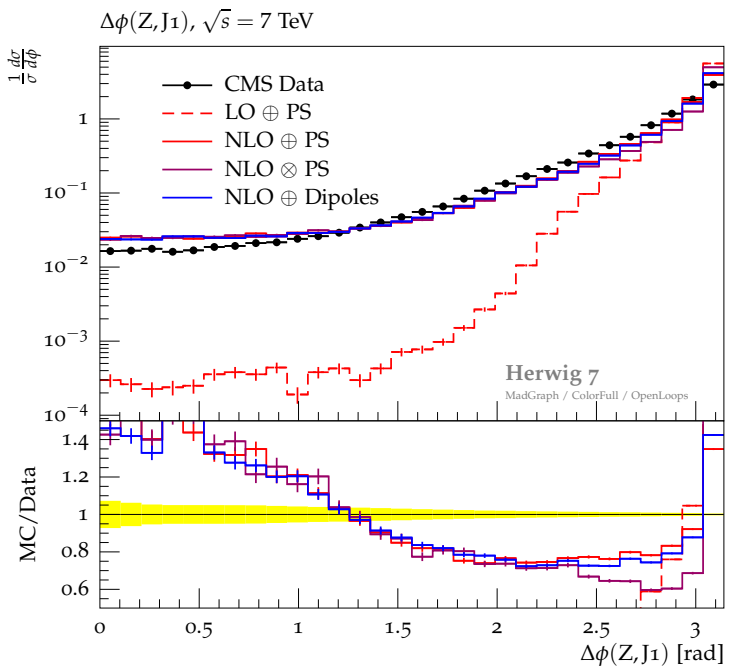

(b)

Figure 4: Examples of HERwig 7 comparisons with data [18], including the new tune, (a) for the $1-T$ distribution in $\mathrm{e}^{+} \mathrm{e}^{-}$and $(b)$ the azimuthal angle between a $Z^{0}$ and its hardest recoil jet. 


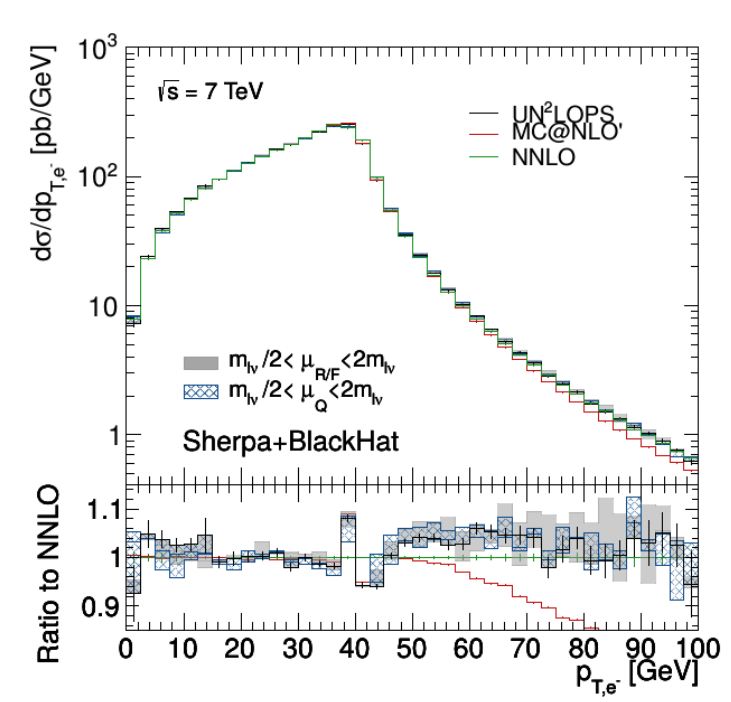

(a)

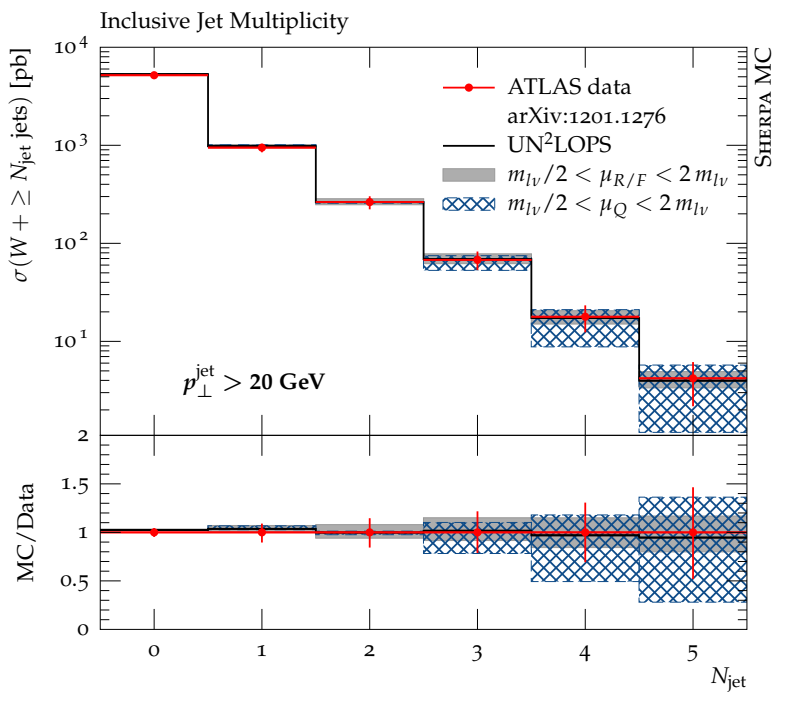

(b)

Figure 5: SHERPA results at NNLO+PS for $\mathrm{W}^{-}$production. (a) Lepton $p_{\perp}$ spectrum [32]. (b) Inclusive jet multiplicity distribution [35].

tarized merging ideas. Other features under development include NLO handling of loop-induced processes, an extended UFO Feynman rules [28] support, extended handling of event reweighting from weight vectors in HEPMC files, improved top decays in the dipole shower, an interface to the HEJ program, and new models for soft interactions and diffraction. The already existing code will also be used for numerous physics studies, to explore and highlight the new possibilities [29].

Noteworthy is that HeRwig 7 has grown to a size of more than 500,000 lines of code. This, as well as the scope of the code, e.g. as manifested by the MATCHBOX module, has exceeded original expectations. In the longer run there is therefore a need for a significant restructuring, although nowhere near the step between HeRwig 6 and HeRwig 7. There are also many ideas for future projects, like amplitude-based parton showers where more interference information can be utilized, e.g. subleading colour contributions [30].

\section{SHERPA news}

SHERPA is undergoing strong but smooth evolution. The latest published manual, for SHERPA 1.1, is already some years old [31], but the SHERPA homepage contains an extensive manual for the current SHERPA 2.2.1 version, along with other documentation and source code.

A significant step is that SHERPA is the first major generator to combine processes at NNLO with parton showers, for $\mathrm{W}^{ \pm}, \gamma^{*} / \mathrm{Z}^{0}$ and $\mathrm{H}^{0}$ production [32, 33]. Examples of results are found in figure 5, showing excellent agreement with data. One-loop matrix elements were obtained from the BLACKHAT library [34], and different multiplicities were combined using the new $\mathrm{UN}^{2} \mathrm{LOPS}$ scheme, wherein unitarity arguments play a key role.

A new dipole shower, DIRE, is available as an option to the existing one [36]. It will be described further in section 8 . 


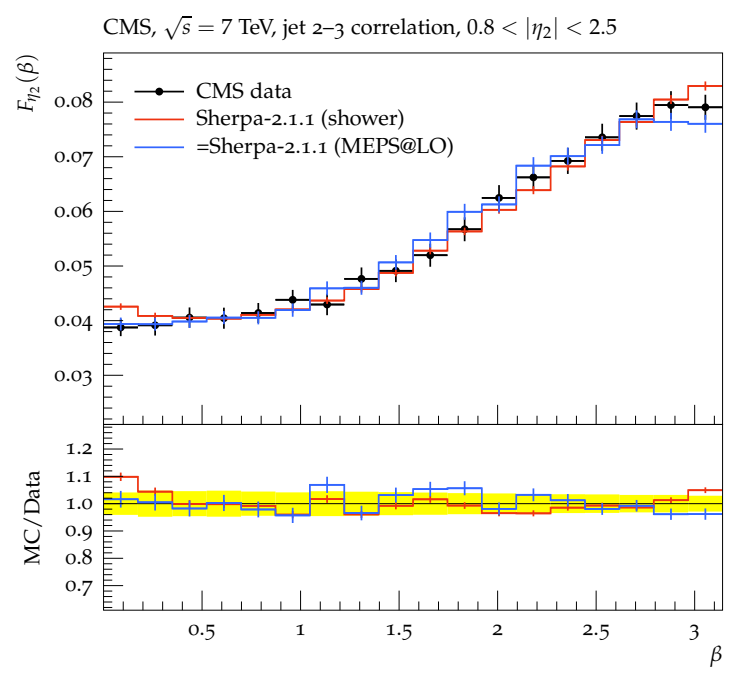

(a)

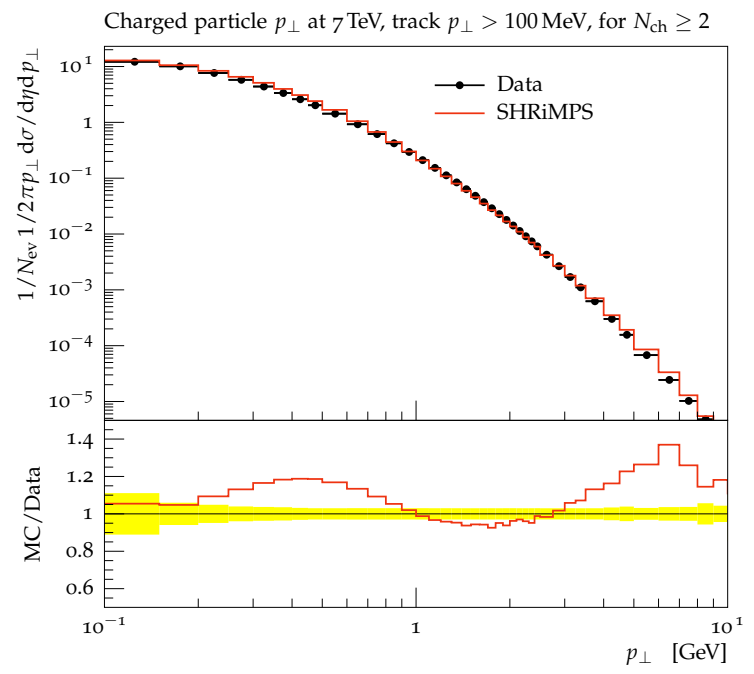

(b)

Figure 6: (a) Coherence angle distribution [35], see [41] for definition . (b) Inclusive charged particle $p_{\perp}$ spectrum in the SHRIMPS framework [43].

Another new feature is a fast way to evaluate uncertainties from the choice of $\alpha_{s}\left(M_{\mathrm{Z}}\right)$, PDF, renormalization and factorization scales, and inherent shower algorithm choices [37]. The shower reweighting will be discussed in section 7. The matrix element part is carried out at NLO, where real emission and one-loop corrections are separated using Catani-Seymour dipole subtraction [23]. The MCGRID package [38] allows for fast and flexible calculation on interpolation grids.

Electroweak (EW) NLO corrections have been included $[39,40]$ for $\mathrm{W} / \mathrm{Z}$ production. These are the first steps towards an automatization of full NLO QCD + EW corrections, combined with showers. Another area of study is corrections for loop-induced processes.

Several physics studies are steadily performed. As an example, coherence in QCD events, e.g the angular distribution of a third soft jet around the second harder jet, is not so well described in HeRwig and Pythia [41], but comes out very well for SHERPA already from the pure shower, figure $6 a$.

The current PYTHIA-inspired MPI framework will eventually be replaced by the SHRIMPS implementation of the Khoze-Martin-Ryskin model [42]. A tuning effort is underway [43], and the current status is exemplified in figure $6 b$.

The key aim of ongoing work is to achieve full NNLO QCD + NLO EW accuracy, combined with showers. These showers will go beyond the current leading-log framework, to include oneloop corrections, which also implies new $1 \rightarrow 3$ splitting kernels (or $2 \rightarrow 4$ in dipole language) and subleading colour corrections. There are also studies to automatize $N$-jettiness slicing [44] to allow more effective generation.

\section{Pythia news}

Pythia 8.2 was released two years ago [45], but represented a rather smooth upgrade from 8.1, which however was a major rewrite from the Fortran-based 6.4 [46]. New subversions are re- 


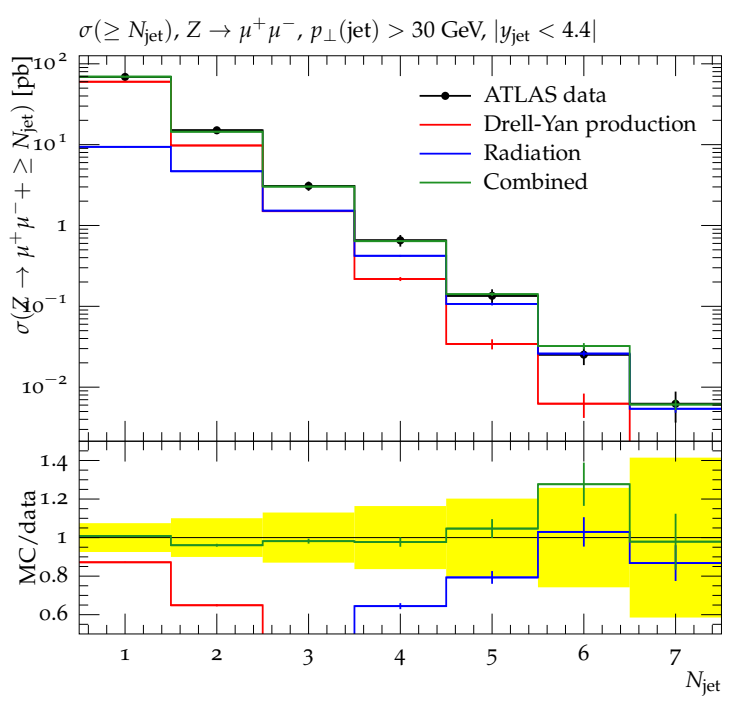

(a)

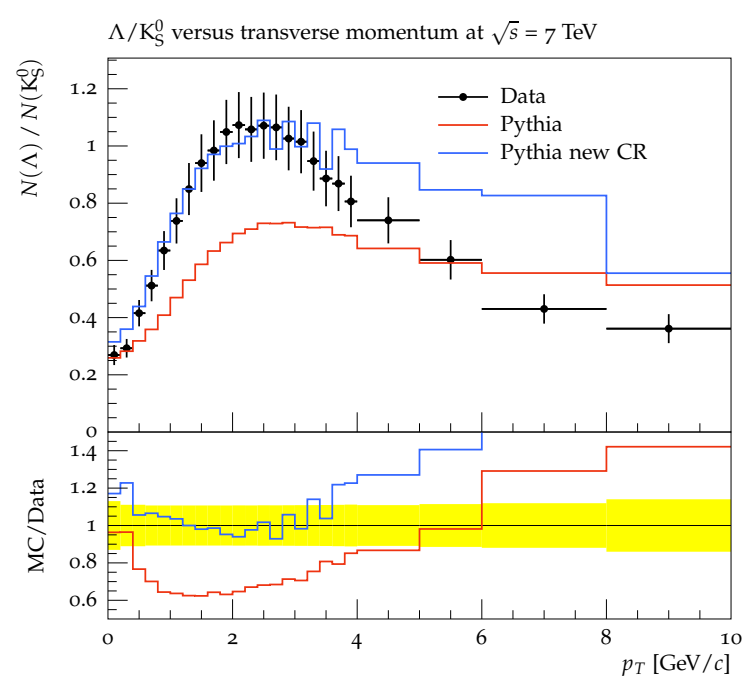

(b)

Figure 7: (a) The inclusive $\mathrm{Z}^{0}+n$-jet rate [50]. (b) The $\Lambda / \mathrm{K}_{S}^{0}$ production ratio as function of $p_{\perp}$ [52].

leased $\sim 3$ times per year, the most recent being 8.219. The PYTHIA homepage contains downloads and full documentation.

The code is distributed with a number of different M\&M methods that can operate on external ME input, e.g. from MAdGrAPH5_AMC@NLO [47] or the PowHeg Box [48], and match those to the internal parton showers. The newest addition to this list is the FxFx method [49].

The shower algorithm has been extended to also include weak branchings, $\mathrm{q} \rightarrow \mathrm{qZ}^{0}$ and $\mathrm{q} \rightarrow$ $\mathrm{q}^{\prime} \mathrm{W}^{ \pm}[50]$. The branching kernels are corrected to representative $s$ - and $t$-channel MEs to obtain a realistic behaviour across the full phase space. The well-known shortfall in the $\mathrm{Z}+n$-jet rate, when only QCD emission off a $\mathrm{q} \overline{\mathrm{q}} \rightarrow \mathrm{Z}^{0}$ base configuration is considered, is well compensated by $\mathrm{Z}^{0}$ radiation off a QCD process, figure $7 a$. The new branchings also offer improvements for $\mathrm{M} \& \mathrm{M}$ strategies [51].

The shower has also been extended to support scale variation evaluation and reweighting of rare shower branchings. The interface for external shower plugins (used e.g. by VINCIA and DIRE) has been extended, such that the internally implemented M\&M schemes can be used also by these.

Many new colour reconnection models have been introduced, in addition to the default one. These have been used to explore features of normal LHC QCD events, e.g. with respect to baryon production properties [52], figure 7b, top mass uncertainties [53] and uncertainties in measurements of $\mathrm{W}^{ \pm}$and $\mathrm{H}^{0}$ properties at a future $\mathrm{e}^{+} \mathrm{e}^{-}$collider [54].

While not part of PYTHIA development efforts, it is worth noting that a recent article [55] makes considerable progress in the long-standing problem of relating the PYTHIA top mass to more traditional pole or $\overline{\mathrm{MS}}$ definitions.

Among other physics changes, a new model for hard diffraction is available [56] and double onium production has been implemented. Several new tunes from the ATLAS and CMS collaborations are easily available, but the Monash one [57] is default. On the technical side, LHEF v3 [58] is now fully supported, it is possible to run the MADGRAPH5_AMC@NLO and POwHEG BOX 
programs from within PyTHIA, and a new interface allows a Python main program to access the full Pythia functionality.

Ongoing work and plans for the future include implementations of $\gamma \gamma, \gamma p$ and ep collisions, new expressions for total, elastic and diffractive cross sections, and alternative hadronization schemes.

\section{Common themes}

Although the three generators are developed independently, and are fully separate codes, there are common trends in their evolution. This comes both from mutual influences and from evolution in the particle physics field as a whole. The most obvious example is that matching and merging strategies have dominated the drive towards higher accuracy for the last 15 years or so. An important precondition is the greatly increased calculational capabilities, e.g. for automated NLO calculations, available through a wide selection of programs. But, starting from this platform, many persons have contributed with schemes to combine matrix elements and parton showers as effectively as possible. The evolution is still very much under way, and there is not one commonly agreed method that does it all. The ongoing debate is very intense and healthy, and it would be impossible to cover it in any detail here. Still, with the risk of doing injustice, here follows a very brief layman's summary.

Historically, two different tasks were addressed separately. One is multileg merging, figure $8 a$. The task there is to combine LO expressions for different parton multiplicities: $n, n+1, n+2$, etc., where $n=0$ for $\mathrm{W} / \mathrm{Z}$ production and $n=2$ for QCD jets. If combined brute-force there will be doublecounting, since a higher jet multiplicity contributes to the inclusive sample of lower multiplicities. With showers added, such doublecounting is further increased. The solution is to include Sudakov factors, as an approximation to higher-order virtual corrections. Or, alternatively put, as a manifestation of a balance between real and virtual corrections, where the addition of an extra jet to an event moves it to another jet multiplicity class while keeping the total cross section (approximately) unchanged. Approaches of this kind include CKKW [59], CKKW-L [60], MLM [61] and UMEPS [62].

The other task is to include full higher-order information, to begin with at NLO, figure $8 b$. Here the two traditional approaches are MC@NLO [20] and POWHEG [63, 21]. The key difference is the handling of the $n+1$ term, which is only accurate to LO. In MC@NLO it is retained as is, while in POWHEG it is rescaled by the same factor $K=\sigma_{\mathrm{NLO}} / \sigma_{\mathrm{LO}}$ as the total cross section. It is possible to interpolate smoothly between these options [64].

The next step along the NLO path is to address NNLO, figure $8 b$, and there are also some schemes directly aimed at that. The main thrust of recent studies has been in a seemingly different direction, however, namely the combination of several NLO calculations, the multileg NLO of figure $8 c$. That is, assuming that you have separate NLO calculations e.g. for $n$ and $n+1$ partons, how do you combine them to achieve NLO accuracy for both $n$ and $n+1$ topologies and LO for $n+2$, also when you match them to showers? As it turns out, multileg NLO can often comparatively easily be extended to NNLO, by adjusting the $n$-parton cross section to preserve a known (semi-)inclusive NNLO cross section. Among this broader class of algorithms one may note MENLOPS [65], MINLO [66], MINLO' [67, 68], MEPS@NLO [69], UNLOPS [70], FxFx [49], 


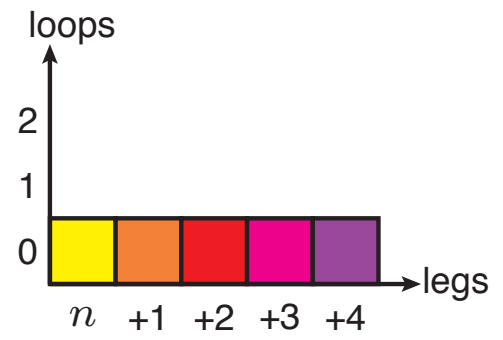

(a)

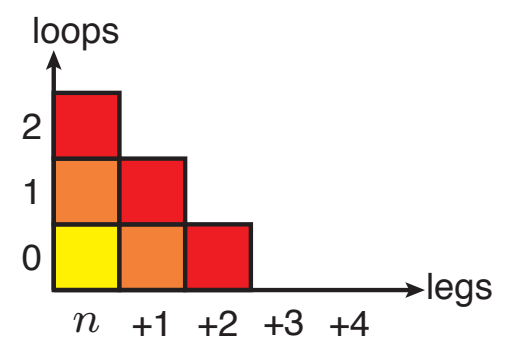

(b)

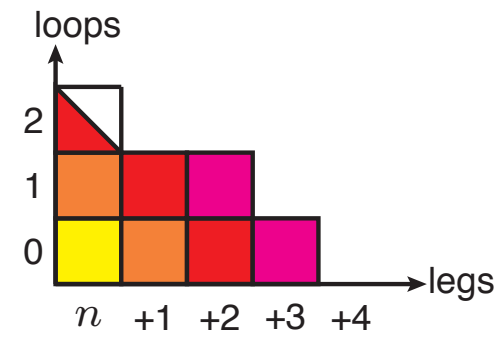

(c)

Figure 8: Three main classes of match and merge strategies. (a) Multileg. (b) Order-by-order. (c) Multileg NLO.

NNLOPS [71], UN²LOPS [32, 33], GENEVA [72], and the Plätzer method [73] further developed for HERWIG 7 [74].

As has already been mentioned, factorization and renormalization scale variations inside the parton showers have been introduced recently both for HERWIG [75], SHERPA [37] and PythiA [76]. The idea is the same: while generating events with the default scale choices, construct alternative event weights, e.g. for a grid of scales. Each weight encodes how likely that shower history would have been, relative to the default one. By suitable reweighting it is thereby feasible to study the scale dependence of any physical observable without having to regenerate and reanalyze events from scratch. It is fairly straightforward to evaluate the part of the total weight that comes from each individual parton branching. The real trick is that the effect of the no-emission Sudakov factors can be obtained from weight factors associated with those trial emissions that fail, when the standard veto algorithm is used to evolve showers.

As a final note, it may be interesting to compare how programs are intended to operate in a world with many external sources of ME input. Here SHERPA is the most restrictive code, that does not accept parton-level LHA/LHEF [77, 58] input from external ME-level generators. All LO MEs are instead calculated internally, while NLO loop corrections are obtained via the BLHA interface [78] or through dedicated interfaces, e.g. to OpEnLoops [79]. It is also possible to provide new ME rules [80] via either UFO [28] or FeynRules [81]. HERWIG requires more ME input and therefore has more external interfaces, e.g. to LHA/LHEF. With the introduction of the MATCHBOX module this communication has become more hidden to the user, however, since MATCHBOX contains dedicated runtime interfaces. Therefore normally also HERWIG should operate as the toplevel program. By contrast, external input is more visible in PyтнiA. There is no built-in ME generator or NLO subtraction code, so whatever is not in the hardcoded library of LO processes has to be obtained from elsewhere, most commonly with LHA/LHEF input. The hierarchy is here less important; e.g. it is possible both to run MADGRAPH5_AMC@NLO from inside PYTHIA and the other way around, but most common is probably to run them separately, with intermediate LHE files stored on disk.

\section{Other generators}

There are many other generators, and we have no intention to list them all. In this section we will briefly mention a few, however, notably those related to the three main generators. 


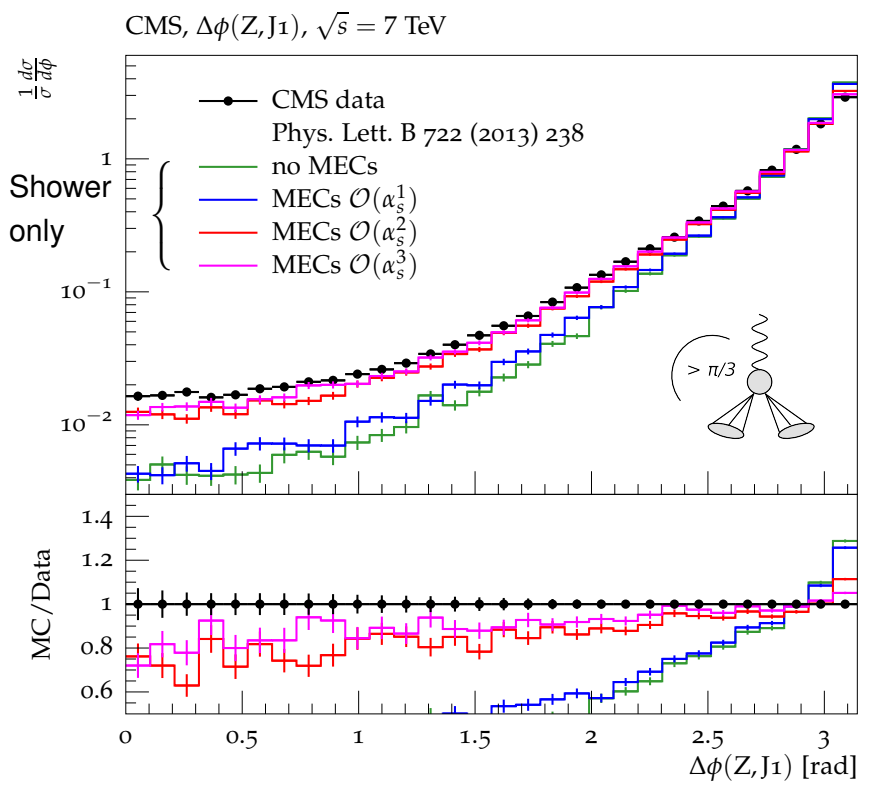

(a)

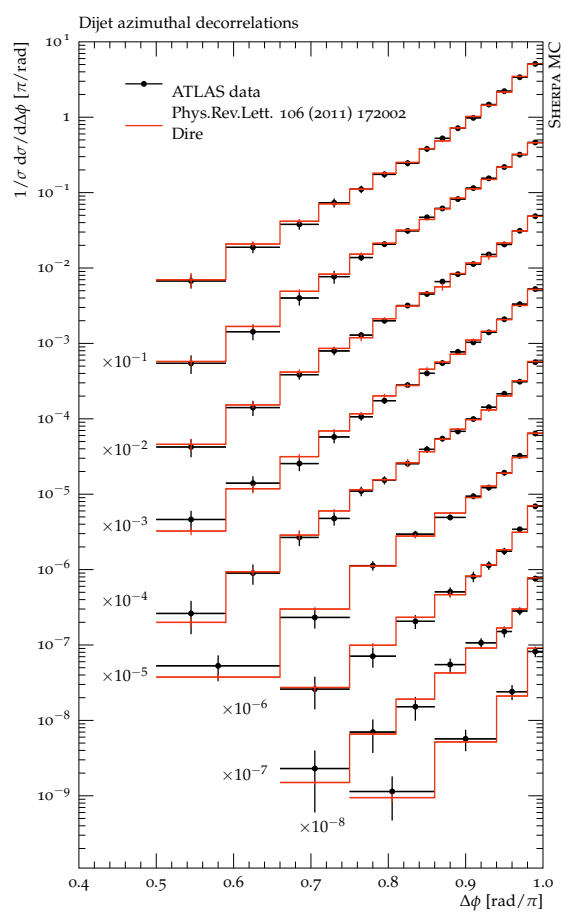

(b)

Figure 9: (a) VINCIA results for the azimuthal angle between a $\mathrm{Z}^{0}$ and the hardest recoil jet [85]. Agreement is further improved when hadronization is added. (b) DIRE results for the dijet azimuthal decorrelation in the SHERPA framework [86].

ARIADNE [82] is the original dipole shower, currently not being developed further on its own, but an important component in some other projects.

VINCIA [83, 84, 85] is a parton shower program, since long for FSR but now also extended to ISR. It is used as a plugin to PYTHIA, replacing the standard showers there. Branchings are defined as $2 \rightarrow 3$ processes with splitting kernels given by antenna functions, which is a specific form of dipole expressions. It has a few unique features. One of them is that it is formulated as a Markovian process, i.e. it retains no memory of the path taken to reach a certain partonic configuration when deciding what to do next. Another is that, after each trial emission, an ME correction factor is applied to bring it into agreement with the full (Born) ME expression to that order (to as high an order as feasible, and of course apart from Sudakov factors). Also, it allows emissions unordered in the $p_{\perp}$ evolution variable, although with a dampened rate. That way the full phase space is covered, without any "dead zones", which is important for the ME corrections to give the desired results. An example how the ME corrections build up order by order is shown in figure $9 a$.

DIRE [86] is unusual in that it is one algorithm implemented as two completely separate codes, one for SHERPA and one for PYTHIA. This has allowed a level of technical checks quite unusual among event generators. (One could wish for more of this, but of course it comes at a price that usually one cannot afford.) It is labelled as a "midpoint between dipole and parton showers", in that it uses the dipole language of $2 \rightarrow 3$ branchings, while the splitting functions single out radiation collinear to individual partons. The evolution variable is, on the other hand, based on transverse 
momentum in the soft limit, and is symmetric in emitter and spectator momentum. The soft terms of the kernels are made less singular by the replacement $1 /(1-z) \rightarrow(1-z) /\left((1-z)^{2}+p_{\perp}^{2} / M^{2}\right)$, where $M$ is the dipole mass. The algorithm is set up to handle negative splitting functions, e.g. from negative PDFs. In the future an extension to NLL is intended, requiring new $1 \rightarrow 3$ splitting kernels and negative corrections to the $1 \rightarrow 2$ ones (cf. NLLJET [87]), so then negative contributions will become more important. An example of comparisons with data is shown in figure $9 b$.

DEDUCTOR $[88,89,90]$ is another parton-shower program, with a strong emphasis on the handling of quantum interference effects, notably in colour and spin. It uses a dipole language, but one where all final partons share the recoil of a branching, whether ISR or FSR. The ordering variable is not a normal $p_{\perp}$ one but based on shower time arguments, which gives a behaviour more like $\left|p^{2}-m^{2}\right| / E$, where the numerator is the parton virtuality.

HEJ (High Energy Jets) [91] provides an all-orders description of processes with more than two jets. It is based on an approximation that should capture hard, wide-angle QCD radiation. As such it is complementary to traditional showers, where the focus is more on soft and collinear emissions. Traditionally it has been combined with the ARIADNE shower, but efforts are underway to interface it to HERWIG and PYTHIA.

GENEVA [72, 92] contains analytic resummation of (up to) NNLL' corrections to NNLO cross sections, using jet resolution criteria such as $N$-jettiness [44] to split the cross section into event classes. It is interfaced to PYTHIA.

There are also many other generators intended to describe heavy-ion physics and/or cosmicray cascades in the atmosphere, such as HiJING [93], DPMJET [94], QGSJET-II [95], and SYBILL [96]. These are all focused on QCD physics aspects, i.e. are not "general-purpose". It would carry too far to describe them in further detail, but a few are especially interesting also for pp applications, and are briefly mentioned here.

EPOS [97] is based on a two-component model. For the collision core a collective hadronization model is used, wherein cluster decays are arranged to produce radial flow, and free parameters are adjusted to be consistent with heavy-ion data, e.g. for the particle composition, thus representing a model for quark-gluon plasma hadronization. For the collision corona instead a stringlike hadronization model is used, akin to traditional pp models. The relative importance of the two components is set event by event based on the collision dynamics. EPOS describes many aspects of LHC soft-physics data better than any of the general-purpose generators, notably collective-flow and QGP-like ones (see further below).

DIPSY $[98,99]$ is a program for dipole evolution in transverse coordinate space, notably for the evolution in the initial state, leading up to the subsequent MPIs. It can be applied both to pp, pA and AA collisions. For the hadronization, an interesting extension is the formation and fragmentation of colour ropes [100], hypothetical objects created when several simple strings combine to form fields of a higher colour representation than the normal triplet one. One disadvantage is that DIPSY is slow to run, especially for $\mathrm{pA}$ and $\mathrm{AA}$, so as an alternative a simpler wounded-nucleon model in the FRITIOF [101] spirit is also made available [102].

\section{Summary and outlook}

Event generators have come a long way over the last forty years, from being a novelty met with 
scepticism to becoming a focal point in much of current-day phenomenological and experimental activities.

The main theme of generator development over the last fifteen years has been to exploit the increasing ME calculational capability, both in terms of more legs and of more loops. The purely perturbative techniques are still limited to a few partons and an even smaller number of loops, and in particular do not fully (or at all) take into account the virtual corrections that are absolutely essential for a physical description. These are the effects that, to some approximation, are encoded in the Sudakov factors of parton showers. To match and merge matrix elements with parton showers thus is not only a matter of improving the showers in the region of hard emissions, it is also a matter of saving the MEs in the soft region where they do not make physical sense on their own. The field is teeming with ideas how to achieve the perfect marriage between MEs and showers. Progress is steadily being made, but we probably are still far from a consensus.

Closely related with this trend, but also relevant on its own merits, is the continued evolution of parton showers. One recent example is the semiautomatic generation of scale-choice uncertainty bands. In the future we are likely to see more emphasis on aspects that go beyond the simple picture of "improved leading log" $1 \rightarrow 2$ or $2 \rightarrow 3$ branchings. This has already begun, e.g. by studies of effects subleading in colour, but will involve many further aspects, such as $\mathscr{O}\left(\alpha_{s}^{2}\right)$ branching kernels to achieve NLL precision.

Somewhat forgotten in this evolution has been soft-physics aspects of the event generation, such as multiparton interactions, colour reconnection, diffraction and hadronization. For a long time we believed in jet universality, i.e. that nonperturbative parton fragmentation basically is the same in $\mathrm{e}^{+} \mathrm{e}^{-}$and pp collisions. This dream has been shattered by LHC data. Most obviously by the observation of the "ridge effect" and other manifestations of collective flow [103, 104, 105]. But even more damning is the increasing fraction of strangeness production for high-multiplicity pp events, in a trend that nicely lines up pp, pA and AA data [106]. Does this imply the (partial) formation of a quark-gluon plasma in high-multiplicity pp collisions, contrary to the standard QGP gospel of a need for larger volumes and time scales than pp can offer? This is the assumption in EPOS, which describes many of these features. Also the colour rope model of DIPSY looks promising. So do either of these offer the correct answer, or what else could be going on? These are burning issues that are not yet reflected in the three standard generators. Clearly much work lies ahead of us in this area, and of a rather less transparent character than in the M\&M game.

Looking ahead, there is the hope that LHC will find new physics, and that the top priority of generator development will be to provide full support for the study of the new processes. If not, it is likely that generator activities will diversify, not only to address technical higher-order precision but also towards a reinvigorated exploration of soft physics.

\section{Acknowledgments}

Work supported in part by the Swedish Research Council, contract number 621-2013-4287, in part by the MCnetITN FP7 Marie Curie Initial Training Network, contract PITN-GA-2012-315877, and in part by the the European Research Council (ERC) under the European Union's Horizon 2020 research and innovation programme, grant agreement No 668679. 


\section{References}

[1] A. Buckley et al., General-purpose event generators for LHC physics, Phys. Rept. 504 (2011) 145 [arXiv:1101.2599 [hep-ph]].

[2] P. Skands, Introduction to QCD, arXiv:1207.2389 [hep-ph].

[3] M. H. Seymour and M. Marx, Monte Carlo Event Generators, arXiv:1304.6677 [hep-ph].

[4] K. A. Olive et al. [Particle Data Group Collaboration], Review of Particle Physics, Chin. Phys. C 38 (2014) 090001, p. 488.

[5] S. Höche, Introduction to parton-shower event generators, arXiv:1411.4085 [hep-ph].

[6] G. Salam, QCD Theory Overview - Towards Precision at LHC, these proceedings.

[7] G. 't Hooft, A Planar Diagram Theory for Strong Interactions, Nucl. Phys. B 72 (1974) 461.

[8] B. Andersson, G. Gustafson and C. Peterson, A Semiclassical Model for Quark Jet Fragmentation, Z. Phys. C 1 (1979) 105.

[9] R. D. Field and R. P. Feynman, A Parametrization of the Properties of Quark Jets, Nucl. Phys. B 136 (1978) 1.

[10] X. Artru and G. Mennessier, String model and multiproduction, Nucl. Phys. B 70 (1974) 93.

[11] B. Andersson, G. Gustafson and T. Sjöstrand, How to Find the Gluon Jets in e+ e-Annihilation, Phys. Lett. B 94 (1980) 211.

[12] W. Bartel et al. [JADE Collaboration], Experimental Study of Jets in electron - Positron Annihilation, Phys. Lett. B 101 (1981) 129.

[13] A. Buckley, J. Butterworth, L. Lönnblad, D. Grellscheid, H. Hoeth, J. Monk, H. Schulz and F. Siegert, Rivet user manual, Comput. Phys. Commun. 184 (2013) 2803 [arXiv:1003.0694 [hep-ph]].

[14] A. Buckley, H. Hoeth, H. Lacker, H. Schulz and J. E. von Seggern, Systematic event generator tuning for the LHC, Eur. Phys. J. C 65 (2010) 331 [arXiv:0907.2973 [hep-ph]].

[15] A. Buckley, J. Ferrando, S. Lloyd, K. Nordström, B. Page, M. Rüfenacht, M. Schönherr and G. Watt, LHAPDF6: parton density access in the LHC precision era, Eur. Phys. J. C 75 (2015) 132 [arXiv:1412.7420 [hep-ph]].

[16] M. Dobbs and J. B. Hansen, The HepMC C++ Monte Carlo event record for High Energy Physics, Comput. Phys. Commun. 134 (2001) 41.

[17] A. Buckley, M. R. Whalley, W. J. Stirling, J. M. Butterworth, E. Nurse and B. Waugh, HepForge: A Lightweight development environment for HEP software, hep-ph/0605046.

[18] J. Bellm et al., Herwig 7.0/Herwig++ 3.0 release note, Eur. Phys. J. C 76 (2016) no.4, 196 [arXiv:1512.01178 [hep-ph]].

[19] M. Bähr et al., Herwig++ Physics and Manual, Eur. Phys. J. C 58 (2008) 639 [arXiv:0803.0883 [hep-ph]].

[20] S. Frixione and B. R. Webber, Matching NLO QCD computations and parton shower simulations, JHEP 0206 (2002) 029 [hep-ph/0204244].

[21] P. Nason, A New method for combining NLO QCD with shower Monte Carlo algorithms, JHEP 0411 (2004) 040 [hep-ph/0409146]. 
[22] S. Plätzer and S. Gieseke, Dipole Showers and Automated NLO Matching in Herwig++, Eur. Phys. J. C 72 (2012) 2187 [arXiv:1109.6256 [hep-ph]].

[23] S. Catani and M. H. Seymour, A General algorithm for calculating jet cross-sections in NLO QCD, Nucl. Phys. B 485 (1997) 291 Erratum: [Nucl. Phys. B 510 (1998) 503] [hep-ph/9605323].

[24] S. Plätzer, Status of Herwig++7, presentation at the ATLAS-CMS Monte Carlo Generators Workshop, 11-12 January 2016, CERN.

[25] M. Cacciari, G. P. Salam and G. Soyez, FastJet User Manual, Eur. Phys. J. C 72 (2012) 1896 [arXiv:1111.6097 [hep-ph]].

[26] S. Gieseke, P. Stephens and B. Webber, New formalism for QCD parton showers, JHEP 0312 (2003) 045 [hep-ph/0310083].

[27] S. Plätzer and S. Gieseke, Coherent Parton Showers with Local Recoils, JHEP 1101 (2011) 024 [arXiv:0909.5593 [hep-ph]].

[28] C. Degrande, C. Duhr, B. Fuks, D. Grellscheid, O. Mattelaer and T. Reiter, UFO - The Universal FeynRules Output, Comput. Phys. Commun. 183 (2012) 1201 [arXiv:1108.2040 [hep-ph]].

[29] M. Rauch and S. Plätzer, Parton Shower Matching Systematics in Vector-Boson-Fusion WW Production, arXiv:1605.07851 [hep-ph].

[30] S. Plätzer and M. Sjödahl, Subleading $N_{c}$ improved Parton Showers, JHEP 1207 (2012) 042 [arXiv:1201.0260 [hep-ph]].

[31] T. Gleisberg, S. Höche, F. Krauss, M. Schönherr, S. Schumann, F. Siegert and J. Winter, Event generation with SHERPA 1.1, JHEP 0902 (2009) 007 [arXiv:0811.4622 [hep-ph]].

[32] S. Höche, Y. Li and S. Prestel, Drell-Yan lepton pair production at NNLO QCD with parton showers, Phys. Rev. D 91 (2015) no.7, 074015 [arXiv:1405.3607 [hep-ph]].

[33] S. Höche, Y. Li and S. Prestel, Higgs-boson production through gluon fusion at NNLO QCD with parton showers, Phys. Rev. D 90 (2014) no.5, 054011 [arXiv:1407.3773 [hep-ph]].

[34] C. F. Berger, Z. Bern, L. J. Dixon, F. Febres Cordero, D. Forde, H. Ita, D. A. Kosower and D. Maitre, An Automated Implementation of On-Shell Methods for One-Loop Amplitudes, Phys. Rev. D 78 (2008) 036003 [arXiv:0803.4180 [hep-ph]].

[35] S. Schumann, Precision simulations for the LHC, presentation at the ATLAS SM Workshop, 1 April 2016, Madrid.

[36] S. Schumann and F. Krauss, A Parton shower algorithm based on Catani-Seymour dipole factorisation, JHEP 0803 (2008) 038 [arXiv:0709.1027 [hep-ph]].

[37] E. Bothmann, M. Schönherr and S. Schumann, Reweighting QCD matrix-element and parton-shower calculations, arXiv:1606.08753 [hep-ph].

[38] E. Bothmann, N. Hartland and S. Schumann, Introducing MCgrid 2.0: Projecting cross section calculations on grids, Comput. Phys. Commun. 196 (2015) 617.

[39] S. Kallweit, J. M. Lindert, P. Maierhöfer, S. Pozzorini and M. Schönherr, NLO electroweak automation and precise predictions for W+multijet production at the LHC, JHEP 1504 (2015) 012 [arXiv:1412.5157 [hep-ph]].

[40] S. Kallweit, J. M. Lindert, P. Maierhofer, S. Pozzorini and M. Schönherr, NLO QCD+EW predictions for $V+$ jets including off-shell vector-boson decays and multijet merging, JHEP 1604 (2016) 021 [arXiv:1511.08692 [hep-ph]]. 
[41] S. Chatrchyan et al. [CMS Collaboration], Probing color coherence effects in pp collisions at $\sqrt{s}=7 \mathrm{TeV}$, Eur. Phys. J. C 74 (2014) no.6, 2901 [arXiv:1311.5815 [hep-ex]].

[42] M. G. Ryskin, A. D. Martin and V. A. Khoze, Soft processes at the LHC. I. Multi-component model, Eur. Phys. J. C 60 (2009) 249 [arXiv:0812.2407 [hep-ph]].

[43] H. Schulz [SHERPA Collaboration], SHRiMPS - Status of soft interactions in SHERPA,

[44] I. W. Stewart, F. J. Tackmann and W. J. Waalewijn, N-Jettiness: An Inclusive Event Shape to Veto Jets, Phys. Rev. Lett. 105 (2010) 092002 [arXiv:1004.2489 [hep-ph]].

[45] T. Sjöstrand et al., An Introduction to PYTHIA 8.2, Comput. Phys. Commun. 191 (2015) 159 [arXiv:1410.3012 [hep-ph]].

[46] T. Sjöstrand, S. Mrenna and P. Z. Skands, PYTHIA 6.4 Physics and Manual, JHEP 0605 (2006) 026 [hep-ph/0603175].

[47] J. Alwall et al., The automated computation of tree-level and next-to-leading order differential cross sections, and their matching to parton shower simulations, JHEP 1407 (2014) 079 [arXiv:1405.0301 [hep-ph]].

[48] S. Alioli, P. Nason, C. Oleari and E. Re, A general framework for implementing NLO calculations in shower Monte Carlo programs: the POWHEG BOX, JHEP 1006 (2010) 043 [arXiv:1002.2581 [hep-ph]].

[49] R. Frederix and S. Frixione, Merging meets matching in MC@NLO, JHEP 1212 (2012) 061 [arXiv:1209.6215 [hep-ph]].

[50] J. R. Christiansen and T. Sjöstrand, Weak Gauge Boson Radiation in Parton Showers, JHEP 1404 (2014) 115 [arXiv:1401.5238 [hep-ph]].

[51] J. R. Christiansen and S. Prestel, Merging weak and QCD showers with matrix elements, Eur. Phys. J. C 76 (2016) no.1, 39 [arXiv:1510.01517 [hep-ph]].

[52] J. R. Christiansen and P. Z. Skands, String Formation Beyond Leading Colour, JHEP 1508 (2015) 003 [arXiv:1505.01681 [hep-ph]].

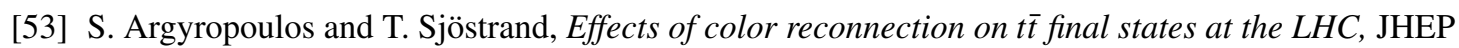
1411 (2014) 043 [arXiv:1407.6653 [hep-ph]].

[54] J. R. Christiansen and T. Sjöstrand, Color reconnection at future $e^{+} e^{-}$colliders, Eur. Phys. J. C 75 (2015) no.9, 441 [arXiv:1506.09085 [hep-ph]].

[55] M. Butenschoen, B. Dehnadi, A. H. Hoang, V. Mateu, M. Preisser and I. W. Stewart, Top Quark Mass Calibration for Monte Carlo Event Generators, arXiv:1608.01318 [hep-ph].

[56] C. O. Rasmussen and T. Sjöstrand, Hard Diffraction with Dynamic Gap Survival, JHEP 1602 (2016) 142 [arXiv:1512.05525 [hep-ph]].

[57] P. Skands, S. Carrazza and J. Rojo, Tuning PYTHIA 8.1: the Monash 2013 Tune, Eur. Phys. J. C 74 (2014) no.8, 3024 [arXiv:1404.5630 [hep-ph]].

[58] J. R. Andersen et al., Les Houches 2013: Physics at TeV Colliders: Standard Model Working Group Report, arXiv:1405.1067 [hep-ph].

[59] S. Catani, F. Krauss, R. Kuhn and B. R. Webber, QCD matrix elements + parton showers, JHEP 0111 (2001) 063 [hep-ph/0109231]. 
[60] L. Lönnblad, Correcting the color dipole cascade model with fixed order matrix elements, JHEP 0205 (2002) 046 [hep-ph/0112284].

[61] M. L. Mangano, M. Moretti, F. Piccinini and M. Treccani, Matching matrix elements and shower evolution for top-quark production in hadronic collisions, JHEP 0701 (2007) 013 [hep-ph/0611129].

[62] L. Lönnblad and S. Prestel, Unitarising Matrix Element + Parton Shower merging, JHEP 1302 (2013) 094 [arXiv:1211.4827 [hep-ph]].

[63] M. Bengtsson and T. Sjöstrand, Coherent Parton Showers Versus Matrix Elements: Implications of PETRA - PEP Data, Phys. Lett. B 185 (1987) 435.

[64] S. Alioli, P. Nason, C. Oleari and E. Re, NLO Higgs boson production via gluon fusion matched with shower in POWHEG, JHEP 0904 (2009) 002 [arXiv:0812.0578 [hep-ph]].

[65] K. Hamilton and P. Nason, Improving NLO-parton shower matched simulations with higher order matrix elements, JHEP 1006 (2010) 039 [arXiv:1004.1764 [hep-ph]].

[66] K. Hamilton, P. Nason and G. Zanderighi, MINLO: Multi-Scale Improved NLO, JHEP 1210 (2012) 155 [arXiv:1206.3572 [hep-ph]].

[67] K. Hamilton, P. Nason, C. Oleari and G. Zanderighi, Merging H/W/Z $+O$ and 1 jet at NLO with no merging scale: a path to parton shower + NNLO matching, JHEP 1305 (2013) 082 doi:10.1007/JHEP05(2013)082 [arXiv:1212.4504 [hep-ph]].

[68] R. Frederix and K. Hamilton, Extending the MINLO method, JHEP 1605 (2016) 042 [arXiv:1512.02663 [hep-ph]].

[69] S. Höche, F. Krauss, M. Schönherr and F. Siegert, QCD matrix elements + parton showers: The NLO case, JHEP 1304 (2013) 027 [arXiv:1207.5030 [hep-ph]].

[70] L. Lönnblad and S. Prestel, Merging Multi-leg NLO Matrix Elements with Parton Showers, JHEP 1303 (2013) 166 [arXiv:1211.7278 [hep-ph]].

[71] K. Hamilton, P. Nason, E. Re and G. Zanderighi, NNLOPS simulation of Higgs boson production, JHEP 1310 (2013) 222 doi:10.1007/JHEP10(2013)222 [arXiv:1309.0017 [hep-ph]].

[72] S. Alioli, C. W. Bauer, C. Berggren, F. J. Tackmann, J. R. Walsh and S. Zuberi, Matching Fully Differential NNLO Calculations and Parton Showers, JHEP 1406 (2014) 089 [arXiv:1311.0286 [hep-ph]].

[73] S. Plätzer, Controlling inclusive cross sections in parton shower + matrix element merging, JHEP 1308 (2013) 114 [arXiv:1211.5467 [hep-ph]].

[74] J. Bellm, S. Gieseke and S. Plätzer, Multijet Merging with the Herwig 7 Dipole Shower, in preparation.

[75] J. Bellm, S. Plätzer, P. Richardson, A. Siodmok and S. Webster, Reweighting Parton Showers, arXiv:1605.08256 [hep-ph].

[76] S. Mrenna and P. Skands, Automated Parton-Shower Variations in Pythia 8, [arXiv:1605.08352 [hep-ph]].

[77] J. Alwall et al., A Standard format for Les Houches event files, Comput. Phys. Commun. 176 (2007) 300 [hep-ph/0609017].

[78] T. Binoth et al., A Proposal for a standard interface between Monte Carlo tools and one-loop programs, Comput. Phys. Commun. 181 (2010) 1612 [arXiv:1001.1307 [hep-ph]]. 
[79] F. Cascioli, P. Maierhofer and S. Pozzorini, Scattering Amplitudes with Open Loops, Phys. Rev. Lett. 108 (2012) 111601 [arXiv:1111.5206 [hep-ph]].

[80] S. Höche, S. Kuttimalai, S. Schumann and F. Siegert, Beyond Standard Model calculations with Sherpa, Eur. Phys. J. C 75 (2015) no.3, 135 [arXiv:1412.6478 [hep-ph]].

[81] N. D. Christensen and C. Duhr, FeynRules - Feynman rules made easy, Comput. Phys. Commun. 180 (2009) 1614 [arXiv:0806.4194 [hep-ph]].

[82] L. Lönnblad, ARIADNE version 4: A Program for simulation of QCD cascades implementing the color dipole model, Comput. Phys. Commun. 71 (1992) 15.

[83] W. T. Giele, D. A. Kosower and P. Z. Skands, A simple shower and matching algorithm, Phys. Rev. D 78 (2008) 014026 [arXiv:0707.3652 [hep-ph]].

[84] W. T. Giele, D. A. Kosower and P. Z. Skands, Higher-Order Corrections to Timelike Jets, Phys. Rev. D 84 (2011) 054003 [arXiv:1102.2126 [hep-ph]].

[85] N. Fischer, S. Prestel, M. Ritzmann and P. Skands, Vincia for Hadron Colliders, arXiv:1605.06142 [hep-ph].

[86] S. Höche and S. Prestel, The midpoint between dipole and parton showers, Eur. Phys. J. C 75 (2015) no.9, 461 [arXiv:1506.05057 [hep-ph]].

[87] K. Kato and T. Munehisa, NLLjet : A Monte Carlo code for e+e-QCD jets including next-to-leading order terms, Comput. Phys. Commun. 64 (1991) 67.

[88] Z. Nagy and D. E. Soper, Parton showers with quantum interference, JHEP 0709 (2007) 114 [arXiv:0706.0017 [hep-ph]].

[89] Z. Nagy and D. E. Soper, A parton shower based on factorization of the quantum density matrix, JHEP 1406 (2014) 097 [arXiv:1401.6364 [hep-ph]].

[90] Z. Nagy and D. E. Soper, Effects of subleading color in a parton shower, JHEP 1507 (2015) 119 [arXiv:1501.00778 [hep-ph]].

[91] J. R. Andersen and J. M. Smillie, Multiple Jets at the LHC with High Energy Jets, JHEP 1106 (2011) 010 [arXiv:1101.5394 [hep-ph]].

[92] S. Alioli, C. W. Bauer, S. Guns and F. J. Tackmann, Underlying event sensitive observables in Drell-Yan production using GENEVA, arXiv:1605.07192 [hep-ph].

[93] M. Gyulassy and X. N. Wang, HIJING 1.0: A Monte Carlo program for parton and particle production in high-energy hadronic and nuclear collisions, Comput. Phys. Commun. 83 (1994) 307 [nucl-th/9502021].

[94] R. Engel and J. Ranft, Hadronic photon-photon interactions at high-energies, Phys. Rev. D 54 (1996) 4244 [hep-ph/9509373].

[95] S. Ostapchenko, QGSJET-II: Towards reliable description of very high energy hadronic interactions, Nucl. Phys. Proc. Suppl. 151 (2006) 143 [hep-ph/0412332].

[96] F. Riehn, R. Engel, A. Fedynitch, T. K. Gaisser and T. Stanev, A new version of the event generator Sibyll, arXiv:1510.00568 [hep-ph].

[97] T. Pierog, I. Karpenko, J. M. Katzy, E. Yatsenko and K. Werner, EPOS LHC: Test of collective hadronization with data measured at the CERN Large Hadron Collider, Phys. Rev. C 92 (2015) no.3, 034906 [arXiv:1306.0121 [hep-ph]]. 
[98] E. Avsar, G. Gustafson and L. Lönnblad, Small-x dipole evolution beyond the large-N(c) imit, JHEP 0701 (2007) 012 [hep-ph/0610157].

[99] C. Flensburg, G. Gustafson and L. Lönnblad, Inclusive and Exclusive Observables from Dipoles in High Energy Collisions, JHEP 1108 (2011) 103 [arXiv:1103.4321 [hep-ph]].

[100] C. Bierlich, G. Gustafson, L. Lönnblad and A. Tarasov, Effects of Overlapping Strings in pp Collisions, JHEP 1503 (2015) 148 [arXiv:1412.6259 [hep-ph]].

[101] B. Andersson, G. Gustafson and B. Nilsson-Almqvist, A Model for Low p(t) Hadronic Reactions, with Generalizations to Hadron - Nucleus and Nucleus-Nucleus Collisions, Nucl. Phys. B 281 (1987) 289.

[102] C. Bierlich, G. Gustafson and L. Lönnblad, Diffractive and non-diffractive wounded nucleons and final states in pA collisions, arXiv:1607.04434 [hep-ph].

[103] V. Khachatryan et al. [CMS Collaboration], Observation of Long-Range Near-Side Angular Correlations in Proton-Proton Collisions at the LHC, JHEP 1009 (2010) 091 [arXiv:1009.4122 [hep-ex]].

[104] G. Aad et al. [ATLAS Collaboration], Observation of Long-Range Elliptic Azimuthal Anisotropies in $\sqrt{s}=13$ and $2.76 \mathrm{TeV}$ pp Collisions with the ATLAS Detector, Phys. Rev. Lett. 116 (2016) no.17, 172301 [arXiv:1509.04776 [hep-ex]].

[105] V. Khachatryan et al. [CMS Collaboration], Evidence for collectivity in pp collisions at the LHC, [arXiv:1606.06198 [nucl-ex]].

[106] J. Adam et al. [ALICE Collaboration], Multiplicity-dependent enhancement of strange and multi-strange hadron production in proton-proton collisions at $\sqrt{s}=7 \mathrm{TeV}$, arXiv: 1606.07424 [nucl-ex]. 